\title{
Susceptibility to ceftobiprole of respiratory-tract pathogens collected in the United Kingdom and Ireland during 20I4-20I5
}

This article was published in the following Dove Press journal: Infection and Drug Resistance

\author{
Anne Santerre Henriksen \\ Jennifer I Smart \\ Kamal Hamed \\ Basilea Pharmaceutica International \\ Ltd., Basel 4005, Switzerland
}

Purpose: Lower respiratory tract infections (LRTIs) can cause significant morbidity and mortality and are becoming increasingly difficult to treat because of the growing prevalence of resistance to conventional antimicrobial agents. This study aimed to assess the current in vitro susceptibility of respiratory tract pathogens collected from the UK and Ireland to ceftobiprole, an advanced-generation cephalosporin, as compared with other antibiotics.

Methods: Pathogens isolated from patients with LRTIs were analyzed as part of the British Society for Antimicrobial Chemotherapy Antimicrobial Resistance Surveillance Programme during 2014-2015. Antibiotic susceptibility was evaluated using European Committee on Antimicrobial Susceptibility Testing breakpoints, including the ceftobiprole pharmacokinetic/ pharmacodynamic non-species-specific breakpoint when species-specific breakpoints were not available.

Results: One thousand one hundred and sixty-eight isolates from community-onset LRTIs and 1,264 isolates from hospital-onset LRTIs were analyzed. The ceftobiprole susceptibility rate was 99.8\% (428/429) for Streptococcus pneumoniae, 100\% (502/502) for Haemophilus influenzae, and 99.6\% (236/237) for Moraxella catarrhalis. All Staphylococcus aureus isolates, including methicillin-susceptible $S$. aureus (MSSA; N=181) and methicillin-resistant $S$. aureus (MRSA; $\mathrm{N}=35$ ), were susceptible to ceftobiprole. Overall, ceftobiprole susceptibility was observed in $88.1 \%$ (215/244) of Escherichia coli isolates, 83.4\% (156/187) of Klebsiella pneumoniae isolates and $86.7 \%$ (98/113) of Enterobacter spp. isolates.

Conclusion: Ceftobiprole had in vitro activity against all $S$. aureus (both MSSA and MRSA) isolates, and almost all $S$. pneumoniae isolates, as well as against Gram-negative bacteria associated with community-onset or hospital-onset LRTIs. Based on this analysis, ceftobiprole is a good treatment option when broad-spectrum antibiotic coverage is needed for LRTIs.

Keywords: antibiotic, BSAC, cephalosporin, MIC, resistance, RTI

\section{Introduction}

Appropriate early empiric treatment is crucial in patients hospitalized for communityacquired lower respiratory tract infections (LRTIs), ${ }^{1}$ and patients with hospital-acquired LRTIs. ${ }^{2}$ Delayed or ineffective therapy in these settings incurs substantial morbidity and mortality. ${ }^{3,4}$ The cephalosporin class of antibiotics is an important component of parenteral treatment algorithms in these settings, ${ }^{1,2,5,6}$ however, as with all antibiotic classes, emerging resistance to broad-spectrum cephalosporins has become a major challenge in the treatment of these infections. ${ }^{7}$ Antibiotic-resistant Gram-positive pathogens, particularly methicillin-resistant Staphylococcus aureus (MRSA), are a significant problem in pulmonary infections. Moreover, significant increases in pneumococcal
Correspondence: Kamal Hamed Basilea Pharmaceutica International Ltd. Grenzacherstrasse 487, Basel 4005, Switzerland

Tel +4I 6I 567 I588

$\mathrm{Fax}+41616061216$

Email kamal.hamed@basilea.com 
resistance to commonly used beta-lactam agents have been documented in recent years. ${ }^{8}$ Empiric selection of an antibiotic treatment with activity against likely causative pathogens is thus complicated not only by the wide range of possible bacteria, but also the growing prevalence of resistance to conventional agents.

Ceftobiprole medocaril is the prodrug of ceftobiprole, a parenteral cephalosporin with in vitro potency against a wide range of respiratory pathogens, including Gram-positive pathogens (methicillin-susceptible $S$. aureus [MSSA], MRSA, pneumococci resistant to penicillin or ceftriaxone) and Gram-negative pathogens (non-extended-spectrum beta-lactamase [ESBL]-producing Enterobacteriaceae, Pseudomonas aeruginosa). ${ }^{9,10}$ S. aureus strains with reduced susceptibility to vancomycin, daptomycin, or linezolid have been shown to remain susceptible to ceftobiprole. ${ }^{11,12}$ The results from randomized, double-blind, Phase III clinical trials have demonstrated that ceftobiprole monotherapy is non-inferior to combination therapy with ceftazidime and linezolid in patients with hospital-acquired pneumonia (HAP), ${ }^{13}$ and non-inferior to ceftriaxone either as monotherapy or in combination with linezolid in community-acquired pneumonia (CAP). ${ }^{14}$ Ceftobiprole is currently approved in around 20 European and non-European countries for the treatment of HAP (excluding ventilator-associated pneumonia [VAP]) and CAP in adults.

The mechanisms underlying development of resistance to cephalosporins are complex and vary among organisms. The main resistance mechanisms include mutation of the target genes (ie, genes encoding penicillin binding proteins [PBPs]), production of hydrolyzing enzymes (ie, beta-lactamases), and overexpression of multidrug efflux pumps. ${ }^{15-18}$ In order to circumvent resistance to beta-lactams, ceftobiprole was specifically designed to have a high affinity for both PBP $2 \mathrm{a}^{19,20}$ of staphylococci, which confers the MRSA phenotype, and PBP2x of Streptococcus pneumoniae strains resistant to penicillin and ceftriaxone. ${ }^{21,22}$ In addition, ceftobiprole has been shown to bind to and inhibit most of the essential PBPs in both Gram-positive and Gram-negative pathogens. ${ }^{23}$ Although ceftobiprole has been shown to have a low propensity for resistance development, ${ }^{24,25}$ regular monitoring of local ceftobiprole susceptibility rates is essential for informed therapy.

The British Society for Antimicrobial Chemotherapy (BSAC) Respiratory Resistance Surveillance Programme is designed to provide long-term surveillance data based on the in vitro activity of a range of antimicrobial agents against potential pathogens isolated from patients with LRTIs in the UK and Ireland. Data collected from the surveillance program during 2014-2015 were reviewed to assess the susceptibility of pathogens from LRTIs to ceftobiprole and other antimicrobial agents.

\section{Methods}

\section{Isolate collection}

The BSAC Respiratory Surveillance Programme is a collection of bacterial isolates provided by 40 sentinel laboratories in the UK and Ireland with wide geographical coverage and diversity between teaching vs non-teaching hospitals, urban vs rural settings, and more vs less socially deprived areas. The current analysis included isolates obtained during surveillance between October 2014 and October 2015.

As described in detail elsewhere, ${ }^{26,27}$ each participating laboratory collected isolates annually (1 October-30 September). Isolates were from lower respiratory samples obtained from patients with community-onset or hospitalonset LRTIs, excluding patients with cystic fibrosis. Patients who tested positive for S. pneumoniae, Haemophilus influenzae, or Moraxella catarrhalis were considered to have community-onset infections, unless they had been admitted to the hospital $>48$ hours before sampling, in which case they were excluded from the analysis. A patient was classified as having a hospital-onset infection if they had been admitted to the hospital $>48$ hours before sampling and had respiratory isolates of $S$. aureus, Pseudomonas spp., Acinetobacter spp., or Enterobacteriaceae. If the pathogen identified was not included in these predefined lists, it was excluded from the analysis (eg, an S. aureus isolate identified in a patient with a community-onset infection would be excluded).

Each center collected up to 14 consecutive isolates of $S$. pneumoniae and $H$. influenzae, up to seven consecutive isolates of $M$. catarrhalis, S. aureus, Pseudomonas spp., Acinetobacter spp., and 28 consecutive isolates of Enterobacteriaceae. Repeat isolates, defined as isolates of the same species obtained from the same patient within 14 days, were assumed to be from the same episode of infection and were excluded. It is important to note that isolates were not linked to a patient identifier and as such, samples from the same patient taken $>14$ days apart and isolates from polymicrobial infections in the same patient were considered separately. Therefore, patient numbers may include duplicate patients where multiple isolates were obtained from the same patient.

Isolates were frozen at $-70^{\circ} \mathrm{C}$ in blood glycerol broth or other established methods and sent to the central laboratory (Public Health England, Colindale, London) for further analysis. 


\section{Microbiological testing}

H. influenzae, M. catarrhalis, S. aureus, Pseudomonas spp., and Enterobacteriaceae spp. other than Escherichia coli were identified using matrix-assisted laser desorption time-offlight mass spectrometry (MALDI-ToF MS). E. coli were identified as pink colored colonies on chromogenic media. S. pneumoniae were identified using optochin and bile solubility tests, followed by either classical serotyping or serotype prediction from genomic sequence data. Acinetobacter baumannii were identified using blaOXA-51 polymerase chain reaction; all other Acinetobacter spp. were identified using MALDI-ToF MS. Minimum inhibitory concentrations (MICs) of ceftobiprole and comparators were determined by the BSAC agar dilution method. ${ }^{28}$ Categorization of isolates as susceptible/intermediate/resistant was based on susceptibility breakpoints from the European Committee on Antimicrobial Susceptibility Testing (EUCAST), ${ }^{29}$ with which the BSAC breakpoints are harmonized. ${ }^{30}$ When species-specific breakpoints were not available, pharmacokinetic/pharmacodynamic (PK/PD) breakpoints were applied. The EUCAST breakpoints for ceftobiprole susceptibility are $2 \mathrm{mg} / \mathrm{L}$ for S. aureus, $0.5 \mathrm{mg} / \mathrm{L}$ for $S$. pneumoniae, and $0.25 \mathrm{mg} / \mathrm{L}$ for Enterobacteriaceae. For interpretation of ceftobiprole MICs for all other species, the PK/PD non-species-specific breakpoint of $4 \mathrm{mg} / \mathrm{L}$ was used. ${ }^{29}$

Isolates of Enterobacteriaceae with ceftazidime or cefotaxime MICs $\geq 1 \mathrm{mg} / \mathrm{L}$ and isolates of Klebsiella oxytoca with a piperacillin/tazobactam MIC $\geq 128 \mathrm{mg} / \mathrm{L}$ (ie, at or above the susceptibility breakpoint for those antimicrobial agents) were tested for the production of ESBLs by BSAC agar dilution or Etest and then confirmed by polymerase chain reaction for the presence of CTX-M. ${ }^{26}$

\section{Data analysis}

$\mathrm{MIC}_{50}, \mathrm{MIC}_{90}$, and $\mathrm{MIC}$ ranges are presented by pathogen or pathogen group, and according to ESBL-producing phenotype where relevant. No statistical analyses were performed in this descriptive study.

\section{Predicting antibiotic resistance in hospital-onset pneumonia}

In a previous study of ceftobiprole activity against pathogens associated with pneumonia, ${ }^{31}$ the authors conducted an experimental analysis to evaluate the clinical relevance of the in vitro susceptibility findings. In their analysis, the current prevalence of the pneumonia pathogens was estimated using data regarding isolates recovered from patients with HAP (excluding VAP) included in a large randomized, controlled, Phase III trial of ceftobiprole medocaril vs ceftazidime plus linezolid. ${ }^{13}$ The data from the Phase III study were then cross-referenced to the susceptibility data obtained from the surveillance study to estimate the proportion of current clinical isolates that would be susceptible to ceftobiprole. In this study, this approach was replicated by cross-referencing the collected susceptibility data with isolate prevalence data reported for HAP/hospital-acquired LRTIs by the European Center for Disease Prevention and Control (ECDC) in its European point prevalence survey of healthcare-associated infections. ${ }^{32}$ The data for ceftazidime-vancomycin in the current study were used as a surrogate to reproduce data reported for ceftazidime-linezolid in the previous study, as susceptibility data for linezolid were not available in the current study.

\section{Results \\ Isolate numbers and patient demographics}

In total, 2,440 isolates were collected. The majority of isolates were from patients who were male and aged 60 years or older (Table 1). The distribution of pathogens based on central laboratory identification is summarized in Table 1 and reflects the collection design. Overall, $47.9 \%$ of isolates $(1,168 / 2,440)$ were from community-onset LRTIs, $51.8 \%$ of isolates $(1,264 / 2,440)$ were from hospital-onset LRTIs (ie, collected from patients hospitalized for $>48$ hours), and $0.3 \%$ of isolates $(8 / 2,440)$ were excluded from the analysis due to missing information or failing to meet the inclusion criteria. Of the isolates collected from patients with hospital-onset LRTIs, 36.4\% $(460 / 1,264)$ were from patients in the intensive care unit (ICU).

\section{In vitro activity of ceftobiprole against community-onset pneumonia pathogens}

In total, 429 S. pneumoniae isolates were assessed, of which 99.8\% (428/429) were susceptible to ceftobiprole (MIC $\leq 0.5 \mathrm{mg} / \mathrm{L}$, with $\mathrm{MIC}_{50 / 90} 0.008 / 0.06 \mathrm{mg} / \mathrm{L}$ ) (Table 2). The only remaining isolate had a ceftobiprole MIC of $1 \mathrm{mg} / \mathrm{L}$. S. pneumoniae demonstrated susceptibility rates of $97.9 \%$ with cefotaxime, but $\leq 84.6 \%$ for the other agents tested, with $83.7 \%$ of isolates susceptible to penicillin (Table 3 ). No isolates of $S$. pneumoniae were inhibited by ciprofloxacin.

Using the PK/PD breakpoint, 100\% (502/502) H. influen$z a e$ isolates were susceptible to ceftobiprole, as were $99.6 \%$ (236/237) of the M. catarrhalis isolates ( $\mathrm{MIC}_{50 / 90} 1 / 4 \mathrm{mg} / \mathrm{L}$ ) (Table 2). 
Table I Patient and isolate characteristics

\begin{tabular}{|c|c|}
\hline & $\mathbf{N}(\%)$ \\
\hline Total number of isolates ${ }^{a}$ & 2,440 \\
\hline Gender, male & $\mathrm{I}, 426(58.4)$ \\
\hline \multicolumn{2}{|l|}{ Age, years ${ }^{b}$} \\
\hline $0-4$ & $110(4.5)$ \\
\hline $5-19$ & $53(2.2)$ \\
\hline 20-39 & $169(6.9)$ \\
\hline $40-49$ & $199(8.2)$ \\
\hline $50-59$ & $301(12.3)$ \\
\hline $60-69$ & $619(25.4)$ \\
\hline 70-79 & $634(26.0)$ \\
\hline$\geq 80$ & $354(14.5)$ \\
\hline \multicolumn{2}{|l|}{ Infection type } \\
\hline Hospital-onset infection & $\mathrm{I}, 264(5 \mathrm{I} .8)$ \\
\hline $\mathrm{ICU}$ & $460(36.4)$ \\
\hline Community-onset infection & $\mathrm{I}, \mathrm{I} 68$ (47.9) \\
\hline Other & $8(0.3)$ \\
\hline \multicolumn{2}{|l|}{ Pathogen (central testing) } \\
\hline Hospital-onset pneumonia & $\mathrm{I}, 264(5 \mathrm{I} .8)$ \\
\hline Escherichia coli & $244(19.3)$ \\
\hline Non-ESBL & 213 \\
\hline ESBL & 31 \\
\hline Staphylococcus aureus & $216(17.1)$ \\
\hline MSSA & 181 \\
\hline MRSA & 35 \\
\hline Pseudomonas aeruginosa & $214(16.9)$ \\
\hline Klebsiella pneumoniae & I87 (|4.8) \\
\hline Non-ESBL & 159 \\
\hline ESBL & 28 \\
\hline Enterobacter spp. & $113(8.9)^{d}$ \\
\hline Klebsiella oxytoca & $63(5.0)$ \\
\hline Serratia spp. & $56(4.4)^{\mathrm{e}}$ \\
\hline Acinetobacter baumannii & $43(3.4)$ \\
\hline Citrobacter spp. & $42(3.3)^{f}$ \\
\hline Community-onset pneumonia & $\mathrm{I}, \mathrm{I} 68(47.7)$ \\
\hline Haemophilus influenzae & $502(43.0)$ \\
\hline Streptococcus pneumoniae & $429(36.7)$ \\
\hline Moraxella catarrhalis & $237(20.3)$ \\
\hline
\end{tabular}

Notes: as multiple isolates were collected from the same patient in a small proportion of patients with prolonged or polymicrobial infections, these values are estimates and are likely to be a slight overestimation of the number of patients included. ${ }^{b}$ Age was not known for one patient. 'Excluded from analysis; includes I $H$. influenzae isolate from a patient who was admitted for $>48$ hours when sample was taken, and 7 isolates for which information was incomplete. ${ }^{\mathrm{d}} 68 \mathrm{E}$. cloacae, 23 E. aerogenes, I 5 E. cloacae complex, $7 \mathrm{E}$. asburiae isolates, and I E. gergoviae isolate. e5I S. marcescens and $4 \mathrm{~S}$. liquefaciens isolates. ${ }^{34} \mathrm{C}$. koseri and $7 \mathrm{C}$. freundii isolates, and I C. braakii isolate.

Abbreviations: ESBL, extended-spectrum beta-lactamases; ICU, intensive care unit; MRSA, methicillin-resistant S. aureus; MSSA, methicillin-susceptible S. aureus.

\section{In vitro activity of ceftobiprole against hospital-onset pneumonia pathogens}

Of the 244 E. coli isolates collected, $87.3 \%(213 / 244)$ were non-ESBL producers and $12.7 \%(31 / 244)$ had an ESBL-positive phenotype. Overall, $88.1 \%(215 / 244)$ of $E$. coli isolates were susceptible to ceftobiprole, similar to the susceptibility rate for ceftazidime $(86.1 \%$; 210/244). Ceftobiprole was active against $98.6 \%(210 / 213)$ of non-ESBL-producing
E. coli isolates, but had reduced activity against ESBLproducing isolates, with only $16.1 \%(5 / 31)$ of such isolates being susceptible to ceftobiprole (Table 2). Only meropenem was active against $100 \%$ of $E$. coli isolates (Table 3 ).

Other Enterobacteriaceae species isolated included Klebsiella pneumoniae and Enterobacter spp. From a total of $187 \mathrm{~K}$. pneumoniae isolates examined, $83.4 \%(156 / 187)$ were susceptible to ceftobiprole. Ceftobiprole was active against $96.9 \%$ (154/159) of non-ESBL producing isolates of K. pneumoniae, while the 28 isolates with an ESBL-positive phenotype had a low rate of susceptibility $(7.1 \%$ [2/28]; 26 of 28 had a ceftobiprole MIC $\leq 2 \mathrm{mg} / \mathrm{L}$ ) (Table 2). The agent with the lowest rate of resistance for $K$. pneumoniae isolates was meropenem, with only $1.1 \%(2 / 187)$ of isolates displaying resistance. Resistance rates to the other agents ranged from $10.7 \%(20 / 187)$ for ceftazidime, gentamicin, and piperacillin/ tazobactam, to $11.8 \%$ (22/187) for ciprofloxacin (Table 3).

A total of 113 Enterobacter isolates were assessed, of which $86.7 \%$ (98/113) were ceftobiprole-susceptible; $\mathrm{MIC}_{50}$ and $\mathrm{MIC}_{90}$ values were 0.06 and $2 \mathrm{mg} / \mathrm{L}$, respectively (Table 2). Meropenem was the only agent with activity against all Enterobacter isolates; resistance rates for the other agents ranged from $1.8 \%(2 / 113)$ for gentamicin to $22.1 \%(25 / 113)$ for ceftazidime (Table 3 ).

Of the $216 \mathrm{~S}$. aureus isolates, $83.8 \%(181 / 216)$ were MSSA and $16.2 \%(35 / 216)$ were MRSA. All $S$. aureus isolates, both MSSA and MRSA, were susceptible to ceftobiprole (MIC $\leq 2 \mathrm{mg} / \mathrm{L}$ ), with $\mathrm{MIC}_{50 / 90}$ values of $0.5 / 0.5 \mathrm{mg} / \mathrm{L}$ for MSSA and $1 / 2 \mathrm{mg} / \mathrm{L}$ for MRSA (Table 2). Among the other antimicrobial agents tested, $100 \%$ of $S$. aureus isolates were also susceptible to vancomycin (MIC $\leq 2 \mathrm{mg} / \mathrm{L}$, with $\left.\mathrm{MIC}_{50 / 90} 1 / 1 \mathrm{mg} / \mathrm{L}\right)$. For ciprofloxacin and erythromycin, resistance was observed in 18.5\% (40/216) and 20.8\% (45/216) of S. aureus isolates, respectively (Table 3 ). For the MRSA isolates, the rate of resistance was $91.4 \%$ (32/35) for ciprofloxacin and $68.6 \%$ (24/35) for erythromycin.

Applying the ceftobiprole PK/PD breakpoint of $4 \mathrm{mg} / \mathrm{L}$, ceftobiprole was active against $86.0 \%(184 / 214)$ of the P. aeruginosa isolates tested (Table 2). The highest rates of susceptibility were seen for colistin $(98.6 \% ; 211 / 214)$ and ceftolozane/tazobactam $(99.1 \% ; 212 / 214)$ (Table 3). Ceftazidime resistance was detected in $7.9 \%$ (17/214) of P. aeruginosa isolates, 9 of which were also ceftobiprole-resistant. Of the 197 ceftazidime-sensitive P. aeruginosa isolates, $10.7 \%$ (21/197) were ceftobiprole-resistant (Table 2). Resistance rates for $P$. aeruginos $a$ against the other agents ranged from $3.7 \%(8 / 214)$ for gentamicin to $13.1 \%$ (28/214) for piperacillin/tazobactam (Table 3). 


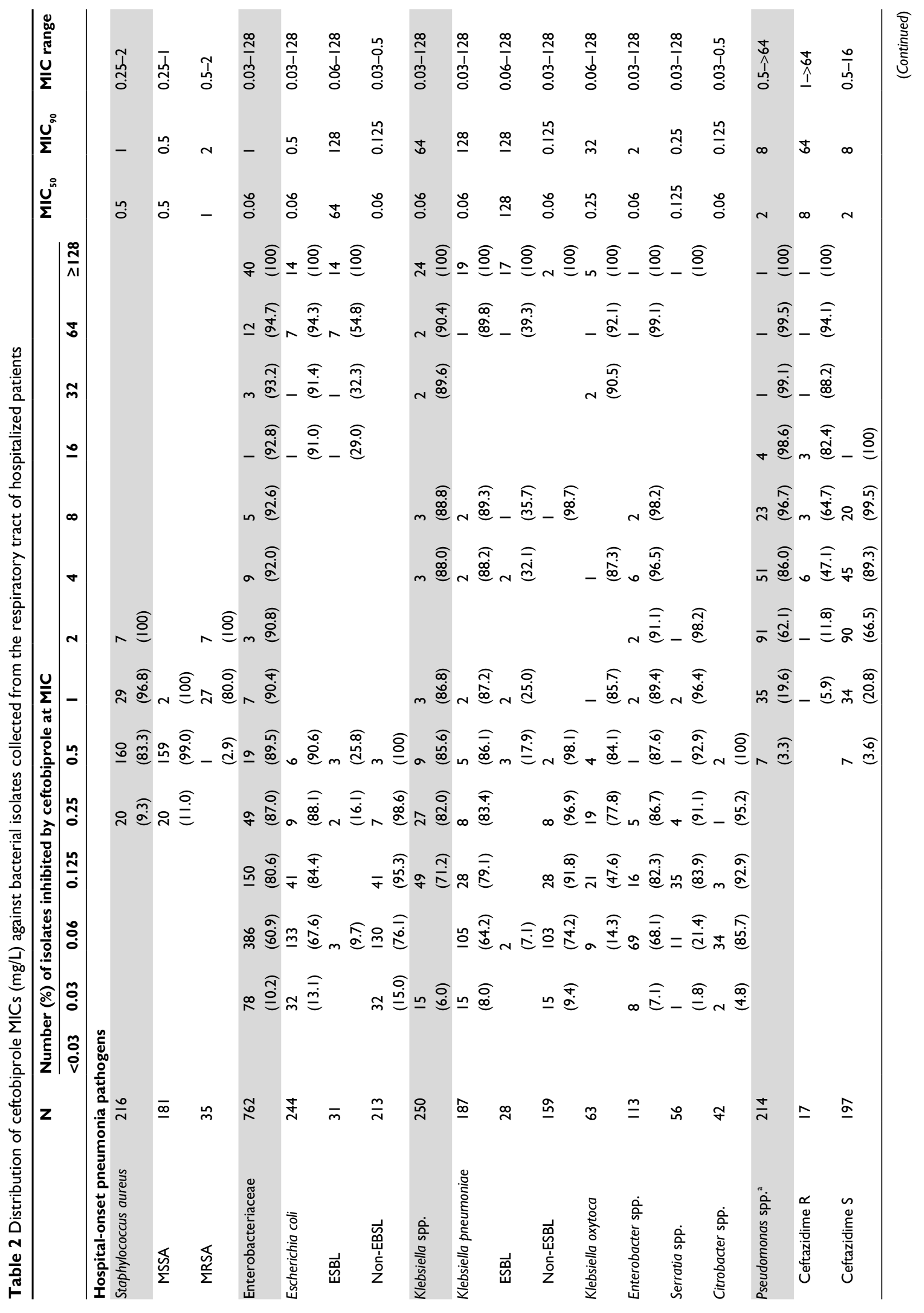




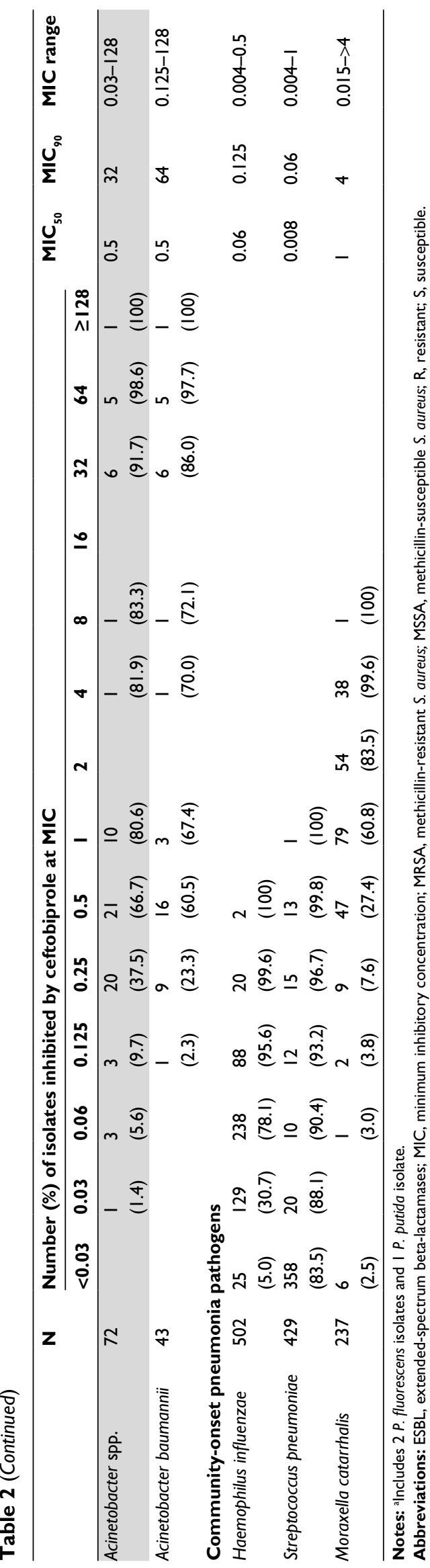

Amongst $A$. baumannii isolates, $69.8 \%$ (30/43) were susceptible to ceftobiprole, with $\mathrm{MIC}_{50 / 90}$ values of $0.5 / 64 \mathrm{mg} / \mathrm{L}$ (Table 2). The susceptibility rates for A. baumannii for meropenem $(69.8 \%$; 30/43), gentamicin $(69.8 \% ; 30 / 43)$, and piperacillin/tazobactam $(65.1 \% ; 28 / 43)$ were similar or identical to the rate observed for ceftobiprole, but there was a low rate of susceptibility $(34.9 \% ; 15 / 43)$ to ceftazidime in this species (Table 3 ).

\section{Predicting antibiotic resistance in hospital-onset pneumonia}

An exploratory analysis was carried out to predict the proportion of healthcare-associated respiratory pathogens in the UK likely to be susceptible to the different antimicrobial agents. Using an approach replicated from a previous study, ${ }^{31}$ susceptibility rates observed in the current study were cross-referenced with prevalence data reported in the ECDC European point prevalence survey of healthcare-associated respiratory infections. ${ }^{32}$

The proportion of susceptible isolates from HAP/ hospital-onset LRTIs in the UK was predicted to be $87.8 \%$ for ceftobiprole, $89.4 \%$ for meropenem, $82.1 \%$ for piperacillin/tazobactam, and $92.9 \%$ for ceftazidime-vancomycin (Table 4).

\section{Discussion}

The data presented here from the BSAC Surveillance Programme during the 2014-2015 season confirm the continued potent activity of ceftobiprole against the key respiratory pathogens associated with community-onset or hospitalonset LRTIs in the UK and Ireland. In CAP, S. pneumoniae, H. influenzae, and M. catarrhalis are the primary causative pathogens. ${ }^{33}$ All H. influenzae isolates were susceptible to ceftobiprole. Susceptibility to ceftobiprole was also high in S. pneumoniae and M. catarrhalis, with susceptibility rates of $99.8 \%$ and $99.6 \%$, respectively. These data suggest that ceftobiprole is a good empirical treatment choice for patients with CAP, given its comprehensive activity against the most common causative Gram-positive and Gram-negative pathogens.

In HAP, S. aureus, P. aeruginosa, and Enterobacteriaceae (including E. coli and K. pneumoniae) account for the majority of infections. ${ }^{32,34}$ Ceftobiprole demonstrated potent in vitro activity against all $S$. aureus isolates tested, including both MSSA and MRSA isolates. Ceftobiprole also showed in vitro activity against S. aureus isolates that were resistant to other antimicrobial agents, such as ciprofloxacin and erythromycin. Only vancomycin demonstrated similar susceptibility rates against all $S$. aureus isolates. 
Table 3 In vitro activity of ceftobiprole and comparators against bacterial isolates collected from the respiratory tract of hospitalized patients

\begin{tabular}{|c|c|c|c|c|}
\hline & \multicolumn{3}{|c|}{ MIC (mg/L) } & \multirow{2}{*}{$\begin{array}{l}\text { \% Susceptible/resistant } \\
\text { EUCAST }^{\mathbf{a}}\end{array}$} \\
\hline & $\mathrm{MIC}_{50}$ & $\mathrm{MIC}_{90}$ & Range & \\
\hline \multicolumn{5}{|c|}{ Hospital-onset pneumonia pathogens } \\
\hline \multicolumn{5}{|c|}{ Staphylococcus aureus $(\mathrm{N}=2 \mid 6)$} \\
\hline Ceftobiprole & 0.5 & 1 & $0.25-2$ & $100 / 0$ \\
\hline Oxacillin & 0.5 & 128 & $0.125->128$ & $83.8 / 16.2$ \\
\hline Tetracycline & 0.5 & 0.5 & $\leq 0.06->128$ & $95.8 / 4.2$ \\
\hline Trimethoprim & 1 & 2 & $0.125->128$ & $92.6 / 7.4$ \\
\hline Vancomycin & I & 1 & $0.5-2$ & $100 / 0$ \\
\hline Ciprofloxacin & 0.5 & 128 & $0.125->128$ & $81.5 / 18.5^{b}$ \\
\hline Erythromycin & 0.5 & $>128$ & $0.125->128$ & $79.2 / 20.8^{c}$ \\
\hline Gentamicin & 0.25 & 0.25 & $0.06-32$ & $97.2 / 2.8$ \\
\hline \multicolumn{5}{|c|}{ Enterobacteriaceae $(\mathrm{N}=762)$} \\
\hline Ceftobiprole & 0.06 & 1 & $0.03-128$ & $87.0 / 13.0$ \\
\hline Ciprofloxacin & 0.03 & 1 & $0.004->256$ & $87.1 / 11.3$ \\
\hline Ceftazidime & 0.25 & 4 & $0.03->256$ & $86.4 / 9.1$ \\
\hline Meropenem & 0.03 & 0.06 & $0.015->256$ & $99.7 / 0.3$ \\
\hline Piperacillin/tazobactam & 2 & 16 & $0.06->256$ & $85.3 / 9.3$ \\
\hline Gentamicin & 0.5 & 1 & $\leq 0.125->256$ & $93.6 / 6.2$ \\
\hline \multicolumn{5}{|c|}{ Escherichia coli $(\mathrm{N}=244)$} \\
\hline Ceftobiprole & 0.06 & 0.5 & $0.03-128$ & $88.1 / 11.9$ \\
\hline Ciprofloxacin & 0.015 & 32 & $0.004-256$ & $78.7 / 20.1$ \\
\hline Ceftazidime & 0.25 & 4 & $0.06-256$ & $86.1 / 7.0$ \\
\hline Meropenem & 0.015 & 0.03 & $0.015-0.06$ & $100 / 0$ \\
\hline Piperacillin/tazobactam & 2 & 16 & $I->256$ & $87.3 / 8.6$ \\
\hline Gentamicin & 0.5 & 4 & $0.25-64$ & $89.7 / 9.4$ \\
\hline \multicolumn{5}{|c|}{ Klebsiella pneumoniae $(\mathbf{N}=\mid 87)$} \\
\hline Ceftobiprole & 0.06 & 128 & $0.03-128$ & $83.4 / 16.6$ \\
\hline Ciprofloxacin & 0.03 & 1 & $0.008->256$ & $85.6 / 11.8$ \\
\hline Ceftazidime & 0.25 & 8 & $0.06->256$ & $84.0 / 10.7$ \\
\hline Meropenem & 0.03 & 0.03 & $0.015 \rightarrow 256$ & $98.9 / 1.1$ \\
\hline Piperacillin/tazobactam & 4 & 32 & $0.5->256$ & $82.4 / 10.7$ \\
\hline Gentamicin & 0.25 & 16 & $\leq 0.125->256$ & $89.3 / 10.7$ \\
\hline \multicolumn{5}{|c|}{ Enterobacter spp. $(\mathrm{N}=|| 3)$} \\
\hline Ceftobiprole & 0.06 & 2 & $0.03-128$ & $86.7 / 13.3$ \\
\hline Ciprofloxacin & 0.015 & 0.06 & $0.008-128$ & $93.8 / 3.5$ \\
\hline Ceftazidime & 0.25 & 64 & $0.125->256$ & $75.2 / 22.1$ \\
\hline Meropenem & 0.03 & 0.125 & $0.015-0.5$ & $100 / 0$ \\
\hline Piperacillin/tazobactam & 4 & 32 & $\mathrm{I}-256$ & $78.8 / 15.0$ \\
\hline Gentamicin & 0.25 & 0.5 & $\leq 0.125-64$ & $98.2 / 1.8$ \\
\hline \multicolumn{5}{|c|}{ Pseudomonas aeruginosa $(\mathrm{N}=214)^{d}$} \\
\hline Ceftobiprole & 2 & 8 & $0.5->64$ & $86.0 / 14.0^{\mathrm{e}}$ \\
\hline Ciprofloxacin & 0.125 & 2 & $0.015-32$ & $85.0 / 15.0$ \\
\hline Ceftazidime & 2 & 8 & $0.25->256$ & $92.1 / 7.9$ \\
\hline Piperacillin/tazobactam & 4 & 32 & $\leq 0.125->256$ & $86.9 / 13.1$ \\
\hline Meropenem & 0.25 & 8 & $\leq 0.03->32$ & $83.2 / 7.0$ \\
\hline Colistin & 1 & 1 & $0.06->32$ & $98.6 / 1.4$ \\
\hline Gentamicin & 1 & 2 & $\leq 0.125-128$ & $96.3 / 3.7$ \\
\hline Ceftolozane/tazobactam & 0.5 & 1 & $\leq 0.06->256$ & $99.1 / 0.9$ \\
\hline \multicolumn{5}{|l|}{ Acinetobacter spp. $(\mathrm{N}=72)$} \\
\hline Ceftobiprole & 0.5 & 32 & $0.03-128$ & $81.9 / 18.1^{\mathrm{e}}$ \\
\hline Ciprofloxacin & 0.25 & 256 & $0.03->256$ & $80.6 / 19.4$ \\
\hline Ceftazidime & 8 & 256 & $I->256$ & $40.3 / 20.8^{e}$ \\
\hline Meropenem & 0.25 & 32 & $0.06-64$ & $80.6 / 18.1$ \\
\hline
\end{tabular}


Table 3 (Continued)

\begin{tabular}{|c|c|c|c|c|}
\hline & \multicolumn{3}{|c|}{ MIC (mg/L) } & \multirow{2}{*}{$\begin{array}{l}\% \text { Susceptible/resistant } \\
\text { EUCAST }^{a}\end{array}$} \\
\hline & $\mathrm{MIC}_{50}$ & $\mathrm{MIC}_{90}$ & Range & \\
\hline Piperacillin/tazobactam & 0.06 & 256 & $\leq 0.015->256$ & $70.8 / 18.1^{\mathrm{e}}$ \\
\hline Gentamicin & 0.25 & $>256$ & $0.03->256$ & $77.8 / 22.2$ \\
\hline \multicolumn{5}{|c|}{ Acinetobacter baumannii $(\mathbf{N}=43)$} \\
\hline Ceftobiprole & 0.5 & 64 & $0.125-128$ & $69.8 / 30.2^{\mathrm{e}}$ \\
\hline Ciprofloxacin & 0.5 & $>256$ & $0.125->256$ & $67.4 / 32.6$ \\
\hline Ceftazidime & 8 & 256 & $I->256$ & $34.9 / 30.2^{\mathrm{e}}$ \\
\hline Meropenem & 0.25 & 64 & $0.125-64$ & $69.8 / 30.2$ \\
\hline Piperacillin/tazobactam & $\mathrm{I}$ & 256 & $0.03->256$ & $62.8 / 30.2^{\mathrm{e}}$ \\
\hline Gentamicin & 0.25 & $>256$ & $0.03 \rightarrow>256$ & $69.8 / 30.2$ \\
\hline \multicolumn{5}{|c|}{ Community-onset pneumonia pathogens } \\
\hline \multicolumn{5}{|l|}{ Haemophilus influenzae $(\mathbf{N}=502)$} \\
\hline Ceftobiprole & 0.06 & 0.125 & $0.004-0.5$ & $100 / 0^{e}$ \\
\hline Amoxicillin & 0.5 & 64 & $0.06->256$ & $74.3 / 25.7$ \\
\hline Ampicillin & 0.5 & 128 & $0.03->256$ & $69.9 / 30.1$ \\
\hline Ciprofloxacin & 0.008 & 0.015 & $\leq 0.00 \mathrm{I}-4$ & $98.8 / 1.2$ \\
\hline Amoxicillin-clavulanate (2:I ratio) & 0.5 & 1 & $0.03-8$ & $97.6 / 2.4$ \\
\hline Cefotaxime & 0.015 & 0.06 & $\leq 0.001-0.5$ & $97.4 / 2.6$ \\
\hline Tetracycline & 0.5 & 0.5 & $0.06-16$ & $96.8 / 1.0$ \\
\hline \multicolumn{5}{|c|}{ Streptococcus pneumoniae $(\mathbf{N}=429)$} \\
\hline Ceftobiprole & 0.008 & 0.06 & $0.004-1$ & $99.8 / 0.2$ \\
\hline Clindamycin & 0.125 & $>128$ & $0.03->128$ & $84.6 / 15.4$ \\
\hline Erythromycin & 0.125 & $>128$ & $0.06->128$ & $78.8 / 21.2$ \\
\hline Cefotaxime & 0.015 & 0.125 & $\leq 0.004-2$ & $97.9 / 0$ \\
\hline Ciprofloxacin & I & 2 & $0.5-64$ & $0.0 / 94.4^{e}$ \\
\hline Penicillin & 0.015 & 0.25 & $0.004-2$ & $83.7 / 0$ \\
\hline Tetracycline & 0.25 & 32 & $0.06-64$ & $81.1 / 18.6^{f}$ \\
\hline \multicolumn{5}{|l|}{ Moraxella catarrhalis $(\mathbf{N}=\mathbf{2 3 7})$} \\
\hline Ceftobiprole & 1 & 4 & $0.015->4$ & $99.6 / 0.4^{\mathrm{e}}$ \\
\hline Amoxicillin-clavulanate (2:I ratio) & 0.06 & 0.25 & $\leq 0.001-0.25$ & $100 / 0$ \\
\hline Cefotaxime & 0.5 & 1 & $0.06-2$ & $98.7 / 0$ \\
\hline Erythromycin & 0.06 & 0.06 & $0.015-0.25$ & $100 / 0$ \\
\hline Tetracycline & 0.5 & I & $0.25-1$ & $100 / 0$ \\
\hline
\end{tabular}

Notes: ${ }^{2}$ Species-related clinical breakpoint set by EUCAST. ${ }^{\circ} 94.3 \%$ of MRSA were resistant to ciprofloxacin. ${ }^{\text {' } 68.6 \%}$ of MRSA were resistant to erythromycin. ${ }^{\text {Includes }}$ 2 P. fluorescens isolates and I P. putida isolate. 'Susceptibility based on the EUCAST pharmacokinetic/pharmacodynamic breakpoint. ${ }^{\prime}$ Based on clinical breakpoint for benzylpenicillin in indications other than meningitis.

Abbreviations: EUCAST, European Committee on Antimicrobial Susceptibility Testing; MIC, minimum inhibitory concentration; MRSA, methicillin-resistant S. aureus.

Rates of resistance in $S$. aureus were as follows: gentamicin, $2.8 \%$; tetracycline, $4.2 \%$; trimethoprim, $10.2 \%$; oxacillin, $16.3 \%$; ciprofloxacin, $20.8 \%$; and erythromycin, $20.8 \%$.

Gram-negative bacteria associated with HAP also showed high susceptibility to ceftobiprole, with rates of $83.4 \%$ and $88.1 \%$ in $K$. pneumoniae and E. coli isolates, respectively. As observed in previous studies, susceptibility to ceftobiprole was notably lower for ESBL-producing strains. The activity of ceftobiprole against $P$. aeruginosa was good (susceptibility rate: $86.0 \%$ ), while activity against $A$. baumannii was moderate (susceptibility rate: $69.8 \%$ ). However, these data are based on the EUCAST non-species-specific PK/PD breakpoint for ceftobiprole, and therefore additional data will be needed to confirm the correlation of these in vitro results with patient outcomes.

Notably, A. baumannii had the lowest ceftobiprole susceptibility rates of any of the bacterial species tested, a trend also observed with the other antimicrobials included in the study, with overall $A$. baumannii susceptibility rates ranging from $69.8 \%$ for ceftobiprole, meropenem, and gentamicin, to $65.0 \%$ for piperacillin/tazobactam and only $35.4 \%$ for ceftazidime. This pattern of reduced antibiotic susceptibility rates for $A$. baumannii isolates has also been observed in other surveillance studies in Europe. ${ }^{35}$

The high rates of susceptibility to ceftobiprole among MSSA, MRSA, and S. pneumoniae isolates observed in 
Table 4 Predicted susceptibility to ceftobiprole and comparator therapies among the six bacterial species most frequently recovered from patients with healthcare-associated pneumonia/lower respiratory tract infections in Europe. Prevalence data were obtained from the ECDC European point prevalence study of healthcare-associated respiratory infections ${ }^{32}$

\begin{tabular}{|c|c|c|c|c|c|c|c|}
\hline & S. aureus & P. aeruginosa & $\begin{array}{l}\text { Klebsiella } \\
\text { spp. }^{\text {a }}\end{array}$ & E. coli & $\begin{array}{l}\text { Acinetobacter } \\
\text { spp. }\end{array}$ & $\begin{array}{l}\text { Enterobacter } \\
\text { spp. }\end{array}$ & Total \\
\hline \multicolumn{8}{|l|}{ Percentage of isolates recovered from } \\
\hline HAP/LRTIs in Europe (ECDC), \% ${ }^{\mathrm{b}}$ & 12.6 & 17.4 & 11.4 & 8.8 & 8.7 & 5.0 & 63.9 \\
\hline Adjusted percentage $^{c}$ & 19.7 & 27.2 & 17.8 & 13.8 & 13.6 & 7.8 & 100 \\
\hline \multicolumn{8}{|l|}{ Ceftobiprole } \\
\hline Susceptible $(\%)^{d}$ & 100 & 86.0 & 82.0 & 88.1 & 81.9 & 86.7 & NA \\
\hline Predicted $\%$ susceptible & 19.7 & 23.4 & 14.6 & 12.2 & II.I & 6.8 & 87.8 \\
\hline \multicolumn{8}{|l|}{ Piperacillin/tazobactam } \\
\hline Susceptible $(\%)^{d}$ & $83.8^{e}$ & 86.9 & 83.6 & 87.3 & 65.0 & 78.8 & NA \\
\hline Predicted \% susceptible & 16.5 & 23.7 & 15.0 & 12.0 & 8.8 & 6.1 & 82.1 \\
\hline \multicolumn{8}{|l|}{ Ceftazidime + vancomycin } \\
\hline Susceptible $(\%)^{d}$ & $100^{f}$ & $92.1^{g}$ & $87.6^{8}$ & $86.1^{g}$ & $34.9^{8}$ & $75.2^{\mathrm{g}}$ & NA \\
\hline Predicted \% susceptible & 19.7 & 25.1 & 15.6 & 11.9 & 4.7 & 5.9 & 92.9 \\
\hline \multicolumn{8}{|l|}{ Meropenem } \\
\hline Susceptible (\%) ${ }^{d}$ & $83.8^{\mathrm{e}}$ & 83.2 & 99.2 & 100 & 80.6 & 100 & NA \\
\hline Predicted \% susceptible & 16.5 & 22.6 & 17.7 & 13.8 & 11.0 & 7.8 & 89.4 \\
\hline \multicolumn{8}{|c|}{ 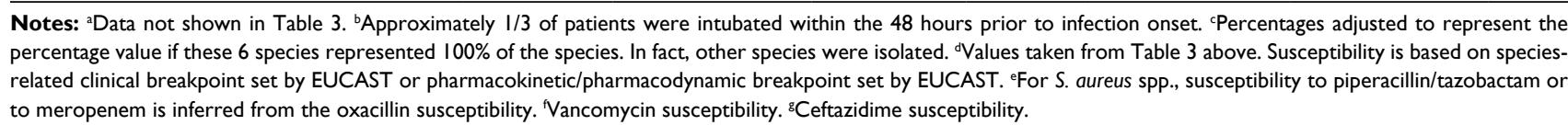 } \\
\hline \multicolumn{8}{|c|}{$\begin{array}{l}\text { Abbreviations: ECDC, European Center for Disease Prevention and Control; EUCAST, European Committee on Antimicrobial Susceptibility Testing; HAP, hospital- } \\
\text { acquired pneumonia; LRTI, lower respiratory tract infection; NA, not applicable. }\end{array}$} \\
\hline
\end{tabular}

this study are similar to ceftobiprole susceptibility rates reported in a previous study of 9,067 pathogens collected from hospitalized patients across the EU and Middle East in 2008 (CLASS study) ${ }^{36}$ In the CLASS study, ceftobiprole susceptibility rates of $100 \%, 99.9 \%$, and $100 \%$ were observed for MSSA, MRSA, and S. pneumoniae, respectively, ${ }^{36}$ which align very closely with the ceftobiprole susceptibility rates observed in the current study $(100 \%, 100 \%$, and $99.8 \%$ for MSSA, MRSA, and S. pneumoniae, respectively). This suggests that the in vitro activity of ceftobiprole against these prevalent Gram-positive pathogens has not changed in almost a decade.

The results of the current study also align well with a recent surveillance study of 12,240 bacterial pathogens collected from Europe, Turkey, and Israel during 2015, in which ceftobiprole susceptibility rates of $100 \%, 96.5 \%$, and 99.3\% were observed for MSSA, MRSA, and S. pneumoniae, respectively. ${ }^{37}$ These results suggest that the activity of ceftobiprole against these pathogens is similar in the UK and Ireland compared with the EU as a whole. It is notable, however, that this same previous study reported a lower rate of susceptibility to ceftobiprole in P. aeruginosa isolates $(70.4 \%)^{37}$ compared with the current study $(86.0 \%$ susceptibility). It is unknown whether this shift is a result of regional susceptibility differences between the UK/Ireland and the EU, as is observed with other cephalosporins,$^{38}$ or as a result of differences in testing methods between the studies; BSAC agar dilution testing was used in the current study, while the Clinical and Laboratory Standards Institute (CLSI) broth microdilution method was used for the previous study.

In another surveillance study by Kresken et al, isolates from respiratory or blood samples from hospitalized patients with pneumonia were collected from 25 laboratories in Austria, Germany, and Switzerland in $2010 .{ }^{31}$ In that analysis, susceptibility to ceftobiprole was similar to the current analysis for MSSA (100\%) and S. pneumoniae (98.8\%). However, only $90.0 \%(27 / 30)$ susceptibility to ceftobiprole was noted among the MRSA isolates, although the significance of this latter finding may be limited due to the low number of isolates assessed. In addition, two of the three non-susceptible isolates were subsequently classified as susceptible when using alternative testing methodology.

To validate the findings of the present study and assess their applicability to clinical practice, an exploratory analysis was conducted, which was designed to estimate the activity of ceftobiprole against the most commonly encountered pathogens causing HAP/hospital-acquired LRTIs in Europe. In this analysis, the susceptibility data presented here from the UK and Ireland were cross-referenced with European pathogen prevalence data in these infections. ${ }^{32}$ European data 
were used due to a lack of available data specific to the UK and Ireland. This analysis predicted that ceftobiprole would be active against $87.8 \%$ of isolates observed in HAP/hospital-acquired LRTIs, which is comparable to the predicted activity of meropenem (89.4\%), and slightly higher than the predicted activity of piperacillin/tazobactam (82.1\%). While this analysis had some limitations, including the assumption that the relative prevalence of causative pathogens observed in HAP in the UK and Ireland is comparable to that observed in Europe, the data support the efficacy of ceftobiprole as an empiric treatment for patients with hospital-acquired respiratory infections in the UK and Ireland and suggest its activity is similar to that of meropenem or piperacillin/tazobactam.

Ceftobiprole may be a valuable treatment option preferable to meropenem or piperacillin/tazobactam in certain countries or healthcare systems that have adopted programs for antibiotic stewardship. These programs often promote carbapenem-sparing strategies to try to reduce the emergence and subsequent spread of antibiotic resistance in Gramnegative pathogens. ${ }^{39,40}$ In the UK, for example, the Commission for Quality and Innovation initiative put in place by NHS England provides financial incentives to reduce the use of meropenem and piperacillin/tazobactam.

Empiric treatment in patients with HAP or hospitalacquired LRTIs is particularly common in the UK, as diagnostic workup is frequently limited (sputum cultures are frequently not representative and bronchial alveolar lavage is too invasive a procedure for most patients); causative respiratory pathogens are thus difficult to obtain and identify. In countries where it is licensed, empiric use of ceftobiprole monotherapy in patients with HAP is also recommended in the 2016 Infectious Diseases Society of America guidelines. ${ }^{2}$ Ceftobiprole may also be a useful option in patients with HAP who are at risk of Gram-negative infection, or at increased risk of mortality, in whom the guidelines recommend combination therapy with antibiotics from two different classes with activity against $P$. aeruginosa.

A limitation in the design of this study is the use of a predefined species collection list. Because of this, the epidemiology of infection in community-onset vs hospital-onset pneumonia could not be investigated. This is a common limitation with antibiotic resistance surveillance studies, which usually feature a similar design to this study. However, an epidemiological analysis of LRTIs was not the key aim of this study. Instead, this study aimed to evaluate the resistance rates against relevant antibiotic agents in a selected range of species known to be commonly associated with LRTIs. The main limitation of the exploratory analysis was the assumption that a comparable prevalence of HAP/hospital-acquired LRTIs would be observed in the UK and Ireland as in the EU.

\section{Conclusion}

As shown by this contemporary data set, ceftobiprole has good in vitro activity against the most clinically relevant Gram-positive and Gram-negative respiratory pathogens, including $P$. aeruginosa and Enterobacter spp. in the UK and Ireland. Therefore, ceftobiprole is an effective alternative option for the empirical treatment of both community- and hospital-onset respiratory infections in the UK and Ireland, due to its broad-spectrum in vitro activity.

\section{Data sharing statement}

The data sets generated and/or analyzed during the current study are available from the corresponding author on reasonable request.

\section{Acknowledgments}

Medical writing support was provided by Eve Blumson of Spirit, Manchester, UK and funded by Basilea Pharmaceutica International Ltd.

Data were collected by BSAC as part of its Respiratory Surveillance Programme and are available online at www. bsacsurv.org.

This work was supported by Basilea Pharmaceutica International Ltd., Basel, Switzerland.

\section{Disclosure}

ASH is a former employee of Basilea Pharmaceutica International Ltd. JIS and KH are employees of Basilea Pharmaceutica International Ltd. The authors report no other conflicts on interest in this work.

\section{References}

1. Mandell LA, Wunderink RG, Anzueto A, et al. Infectious Diseases Society of America/American Thoracic Society consensus guidelines on the management of community-acquired pneumonia in adults. Clin Infect Dis. 2007;44(Supp1 2):S27-S72.

2. Kalil AC, Metersky ML, Klompas M, et al. Management of adults with hospital-acquired and ventilator-associated pneumonia: 2016 clinical practice guidelines by the Infectious Diseases Society of America and the American Thoracic Society. Clin Infect Dis. 2016;63(5): e61-e111.

3. Davey PG, Marwick C. Appropriate vs. inappropriate antimicrobial therapy. Clin Microbiol Infect. 2008;14(Suppl 3):15-21.

4. Lynch JP 3rd. Hospital-acquired pneumonia: risk factors, microbiology, and treatment. Chest. 2001;119(Suppl 2):373S-84S.

5. Bradley JS, Byington CL, Shah SS, et al. Executive summary: the management of community-acquired pneumonia in infants and children older than 3 months of age: clinical practice guidelines by the Pediatric Infectious Diseases Society and the Infectious Diseases Society of America. Clin Infect Dis. 2011;53(7):617-630. 
6. Torres A, Ewig S, Lode H, Carlet J, European HAP working group. Defining, treating and preventing hospital acquired pneumonia: European perspective. Intensive Care Med. 2009;35(1):9-29.

7. Cilloniz C, Martin-Loeches I, Garcia-Vidal C, San Jose A, Torres A. Microbial Etiology of Pneumonia: Epidemiology, Diagnosis and Resistance Patterns. Int J Mol Sci. 2016;17(12):E2120.

8. Jones RN, Sader HS, Moet GJ, Farrell DJ. Declining antimicrobial susceptibility of Streptococcus pneumoniae in the United States: report from the SENTRY Antimicrobial Surveillance Program (1998-2009. Diagn Microbiol Infect Dis. 2010;68(3):334-336.

9. SyedYY. Ceftobiprole medocaril: a review of its use in patients with hospital- or community-acquired pneumonia. Drugs. 2014;74(13):1523-1542.

10. Medicines.org. Zevtera UK Summary of Product Characteristics. Basilea Medical Ltd, Rickmansworth, United Kingdom. Updated 2013. Available from: https://www.medicines.org.uk/emc/product/9164/smpc. Accessed 6 August, 2018.

11. Farrell DJ, Flamm RK, Sader HS, Jones RN. Activity of ceftobiprole against methicillin-resistant Staphylococcus aureus strains with reduced susceptibility to daptomycin, linezolid or vancomycin, and strains with defined SCCmec types. Int J Antimicrob Agents. 2014;43(4):323-327.

12. Mendes RE, Tsakris A, Sader HS, et al. Characterization of methicillinresistant Staphylococcus aureus displaying increased MICs of ceftaroline. J Antimicrob Chemother. 2012;67(6):1321-1324.

13. Awad SS, Rodriguez AH, Chuang YC, et al. A phase 3 randomized double-blind comparison of ceftobiprole medocaril versus ceftazidime plus linezolid for the treatment of hospital-acquired pneumonia. Clin Infect Dis. 2014;59(1):51-61.

14. Nicholson SC, Welte T, File TM, et al. A randomised, double-blind trial comparing ceftobiprole medocaril with ceftriaxone with or without linezolid for the treatment of patients with community-acquired pneumonia requiring hospitalisation. Int J Antimicrob Agents. 2012;39(3): 240-246.

15. Moyá B, Beceiro A, Cabot G, et al. Pan- $\beta$-lactam resistance development in Pseudomonas aeruginosa clinical strains: molecular mechanisms, penicillin-binding protein profiles, and binding affinities. Antimicrob Agents Chemother. 2012;56(9):4771-4778.

16. Lahiri SD, Alm RA. Identification of non-PBP2a resistance mechanisms in Staphylococcus aureus after serial passage with ceftaroline: involvement of other PBPs. J Antimicrob Chemother. 2016;71(11) 3050-3057.

17. Berrazeg M, Jeannot K, Ntsogo Enguéné VY, et al. Mutations in $\beta$-Lactamase AmpC Increase Resistance of Pseudomonas aeruginosa Isolates to Antipseudomonal Cephalosporins. Antimicrob Agents Chemother. 2015;59(10):6248-6255.

18. Fani F, Brotherton MC, Leprohon P, Ouellette M. Genomic analysis and reconstruction of cefotaxime resistance in Streptococcus pneumoniae. J Antimicrob Chemother. 2013;68(8):1718-1727.

19. Hebeisen P, Heinze-Krauss I, Angehrn P, Hohl P, Page MG, Then RL. In vitro and in vivo properties of Ro 63-9141, a novel broad-spectrum cephalosporin with activity against methicillin-resistant staphylococci. Antimicrob Agents Chemother. 2001;45(3):825-836.

20. Farrell DJ, Flamm RK, Sader HS, Jones RN. Ceftobiprole activity against over 60,000 clinical bacterial pathogens isolated in Europe, Turkey, and Israel from 2005 to 2010. Antimicrob Agents Chemother 2014;58(7):3882-3888.

21. Davies TA, Page MG, Shang W, Andrew T, Kania M, Bush K. Binding of ceftobiprole and comparators to the penicillin-binding proteins of Escherichia coli, Pseudomonas aeruginosa, Staphylococcus aureus, and Streptococcus pneumoniae. Antimicrob Agents Chemother. 2007;51(7):2621-2624.

22. Davies TA, Flamm RK, Lynch AS. Activity of ceftobiprole against Streptococcus pneumoniae isolates exhibiting high-level resistance to ceftriaxone. Int J Antimicrob Agents. 2012;39(6):534-538.

23. Hebeisen P, Heinze-Krauss I, Angehrn P, Hohl P, Page MG, Then RL. In vitro and In vivo properties of Ro 63-9141, a novel broad-spectrum cephalosporin with ativity against mthicillin-rsistant saphylococci. Antimicrob Agents Chemother. 2001;45(3):825-836.
24. Chan LC, Basuino L, Diep B, Hamilton S, Chatterjee SS, Chambers HF. Ceftobiprole- and ceftaroline-resistant methicillin-resistant Staphylococcus aureus. Antimicrob Agents Chemother. 2015;59(5):2960-2963.

25. Bogdanovich T, Ednie LM, Shapiro S, Appelbaum PC. Antistaphylococcal activity of ceftobiprole, a new broad-spectrum cephalosporin. Antimicrob Agents Chemother. 2005;49(10):4210-4219.

26. Bsacsurv.org. The British Society for Antimicrobial Chemotherapy Respiratory Resistance Surveillance Project Protocol; 2014. Available from: http://www.bsacsurv.org/wp-content/uploads/2014/09/ PROTOCOL-resp-2013-14-v1-1408.pdf. Accessed 6 August, 2018.

27. Reynolds R, Hope R, Williams L. BSAC Working Parties on Resistance Surveillance. Survey, laboratory, and statistical methods for the BSAC Resistance Surveillance Programmes. JAntimicrob Chemother. 2008;62(Suppl 2):ii15-28.

28. Andrews JM. Determination of minimum inhibitory concentrations. J Antimicrob Chemother. 2001;48(Suppl 1):5-16.

29. Eucast.org. The European Committee on Antimicrobial Susceptibility Testing (EUCAST). Breakpoint tables for interpretation of MICs and zone diameters (version 6.0); 2013. Available from: http://www.eucast. org/clinical_breakpoints/.Accessed 6 August, 2018.

30. Brown DF, Wootton M, Howe RA. Antimicrobial susceptibility testing breakpoints and methods from BSAC to EUCAST. $J$ Antimicrob Chemother. 2016;71(1):3-5.

31. Kresken M, Körber-Irrgang B, Kaase M, et al. In vitro activity of ceftobiprole against key pathogens associated with pneumonia in hospitalized patients: results from the PEG surveillance survey, 2010. GMS Infect Dis. 2015;3:Doc02.

32. Ecdc.europa.eu. European Centre for Disease Prevention and Control. Point prevalence survey of healthcare associated infections and antimicrobial use in European acute care hospitals. Stockholm: ECDC; 2013. Available from: http://ecdc.europa.eu/en/publications/Publications/ healthcare-associated-infections-antimicrobial-use-PPS.pdf. Accessed 6 August, 2018.

33. Cillóniz C, Ewig S, Polverino E, et al. Microbial aetiology of community-acquired pneumonia and its relation to severity. Thorax. 2011;66(4):340-346.

34. Nair GB, Niederman MS. Nosocomial pneumonia: lessons learned. Crit Care Clin. 2013;29(3):521-546.

35. Nowak J, Zander E, Stefanik D, et al. High incidence of pandrug-resistant Acinetobacter baumannii isolates collected from patients with ventilatorassociated pneumonia in Greece, Italy and Spain as part of the MagicBullet clinical trial. J Antimicrob Chemother. 2017;72(12):3277-3282.

36. Rossolini GM, Dryden MS, Kozlov RS, et al. Comparative activity of ceftobiprole against Gram-positive and Gram-negative isolates from Europe and the Middle East: the CLASS study. JAntimicrob Chemother. 2011;66(1):151-159.

37. Pfaller MA, Flamm RK, Duncan LR, et al. Antimicrobial activity of ceftobiprole and comparator agents when tested against contemporary Gram-positive and -negative organisms collected from Europe (2015. Diagn Microbiol Infect Dis. 2018;91(1):77-84.

38. Ecdc.europa.eu. European Centre for Disease Prevention and Control. Surveillance of antimicrobial resistance in Europe. Annual Report of the European Antimicrobial Resistance Surveillance Network (EARS-Net).2016; Stockholm: ECDC. 2017. Available from: https:// ecdc.europa.eu/sites/portal/files/documents/AMR-surveillanceEurope-2016.pdf. Accessed 6 August, 2018.

39. Gov.uk. NHS England Report. English surveillance programme for antimicrobial utilisation and resistance (ESPAUR) 2010 to 2014; 2015. Available from: https://www.gov.uk/government/uploads/system/ uploads/attachment_data/file/477962/ESPAUR_Report_2015.pdf. Accessed 6 August, 2018.

40. Giov.uk. Public Health England. English surveillance programme for antimicrobial utilisation and resistance (ESPAUR) Executive summary and recommendations extracted from 2016 report; 2016. Available from: https://www.gov.uk/government/uploads/system/uploads/attachment_data/file/570276/ESPAUR_executive_summary_and_recommendations.pdf. Accessed 6 August, 2018. 


\section{Publish your work in this journal}

Infection and Drug Resistance is an international, peer-reviewed openaccess journal that focuses on the optimal treatment of infection (bacterial, fungal and viral) and the development and institution of preventive strategies to minimize the development and spread of resistance. The journal is specifically concerned with the epidemiology of antibiotic resistance and the mechanisms of resistance development and diffusion in both hospitals and the community. The manuscript management system is completely online and includes a very quick and fair peerreview system, which is all easy to use. Visit http://www.dovepress.com/ testimonials.php to read real quotes from published authors.

Submit your manuscript here: https://www.dovepress.com/infection-and-drug-resistance-journal 